\title{
Activity and brain amine metabolism in strains of normotensive and hypertensive rats '
}

R. LAVERTY

WELLCOME MEDICAL RESEARCH INSTITUTE, UNIVERSITY OF OTAGO, NEW ZEALAND

Overnight and Y-runway activity was measured in three strains of rats during three months and brain amines and metabolites in various brain regions were then measured. White hypertensive and black rats had less overnight activity and higher levels of noradrenaline, 5-hydroxytryptamine, and total hydroxyindoles than white normotensive animals. Y-runway exploration was not greatly different; white hypertensile rats had a high defecation rate whereas black were low. Renal hypertension had no effect on any activity.

Griew (1966) has shown that rats from a colony with an elevated blood pressure (Smirk \& Hall, 1958) have less spontaneous activity, slower swimming times, and reduced learning ability than normotensive animals from the parent rat colony. These genetic hypertensive rats have a raised level of catecholamines in the brain (Robertson \& Laverty, 1967). In the present paper, the activities of three rat strains, normotensive white, hypertensive white and black, have been observed and the brain levels of catechol and indole amines and metabolites measured in the same rats to determine any correlation of amine metabolism with either blood pressure or activity levels. Another group of rats was made hypertensive by constriction of the renal artery, and the activities of these animals studied to determine the effects of hypertension on activity.

\section{Method}

Male rats, aged three months (approximately $200 \mathrm{~g}$ ) initially, were used throughout. White normotensive rats came from a random-bred albino colony; hypertensive rats, originally selected from the normotensive colony, had been inbred for 18 generations. Black rats were taken from a pure-line colony inbred for 30 generations.

The activity of each group of eight rats was measured in their home cage during three hourly periods on four or six nights.

The animal activity was detected electronically and recorded on paper (Meek, unpublished). Activity traces were assessed against an arbitrary scale and the mean score for each period recorded.

The activity of individual rats, when placed in a Y runway (Steinberg, Rushton, \& Tinson, 1961), was measured fortnightly. The number of entries, rearings, pellets, and the number of bouts of grooming behavior was counted in a $3 \mathrm{~min}$ period.

The brain tissue levels of catecholamines and metabolites were measured in the cerebral cortex, cerebellum, thalamic regions (including the hypothalamus and mid-brain), brain stem (i.e., pons and medulla), and striatum (including some underlying tissue), in each of four rats from each group (Laverty \& Taylor, 1967); 5-hydroxytryptamine and total indoles (Ashcroft \& Sharman, 1962), and homovanillic acid (Juorio, Sharman, \& Trajkov, 1966) were measured fluorimetrically in brain samples from the other four rats from each group.

Renal hypertension was induced in eight white rats (200 $\mathrm{g}$ male) by application of a constriction to the right renal artery. Similar groups of sham-operated and untouched rats served as controls, and activities were recorded over a three month period before and after operation.

\section{Results}

The blood pressure of the white hypertensive group was significantly higher than that of the white normontensive or black groups throughout the experiment.

Nocturnal activities of the groups of rats in their home cages showed some significant differences (Table 1), the white normotensives being more active than the other two groups. Variance analysis of the results showed no significant effect due to cage sensitivity or position. The difference between the strains of rats was less at the end of the experimental period.

Ambulatory activity in the $Y$ runway was not significantly different between the groups, though the hypertensive group showed more rearing. Hypertensives left significantly more fecal pellets whereas black rats left significantly less than the normotensive white rats. Grooming behavior was irregular but there was a statistical difference between hypertensives and both normotensive and black groups.

Table 1 .

Mean blood pressure and activity measurements in groups of 8 white normotensive (N'T), white hypertensive (HT) and black (B) rats

\begin{tabular}{lllll} 
& No. of Observations & NT & HT & B \\
\hline Blood Pressure (mm.Hg) & 6 & 107.5 & $160.2^{* * 1}$ & 105.5 \\
Nocturnal Activity & & & & \\
Age 3-4 months & 4 & 16.9 & $10.7^{* *}$ & $11.7^{* *}$ \\
Age 7-8 months & 4 & 16.6 & $13.9^{*}$ & \\
" & 6 & 11.9 & & 11.7 \\
Y-runway & & & & \\
Entries & 7 & 5.8 & 6.1 & 6.3 \\
Rearing & 7 & 3.3 & $10.1^{1}$ & 3.2 \\
Pellets & 7 & 0.9 & $2.2^{* * 1}$ & $0.0^{* *}$ \\
Grooming & 7 & 0.8 & $0.2^{* * 1}$ & 0.7 \\
\hline
\end{tabular}

\footnotetext{
* Sirmificant difference irom NTP 0.01

* Sionificent difference from NTP $、 0.05$

${ }^{\prime}$ Similicant dilfsernce from $B \quad P$. 0.01
} 
Table 2. Tissue content $(\mu \mathrm{g} / \mathrm{g})$ of amines and metabolites in brain regions (mean of four rats) of white normatensive (NT), white hypertensive (HT) and black (B) rats.

\begin{tabular}{llll} 
& Cortex & Cerebellum & Thalamic Regions \\
\hline $\begin{array}{c}\text { Noradrenaline } \\
\text { NT }\end{array}$ & 0.24 & 0.23 & \\
HT & $0.31^{* *}$ & $0.31^{* * *}$ & 0.58 \\
B & $0.32^{*}$ & 0.25 & $0.65^{*}$ \\
5-hydroxytryptamine & & & $0.71^{*}$ \\
NT & 0.10 & 0.10 & \\
HT & 0.08 & 0.10 & 0.26 \\
B & 0.09 & 0.13 & $0.40^{*}$ \\
Total hydroxyindoles & & $0.31^{*}$ \\
NT & 0.82 & 0.46 & 1.16 \\
HT & 0.86 & $0.63^{* *}$ & $1.47^{*}$ \\
B & 0.82 & $0.54^{*}$ & $1.41^{* *}$ \\
\hline
\end{tabular}

*** Significant difference from $N T \quad P<0.001$

** Significant difference from $N T P<0.01$

* Significant difference from $N T P<0.05$

Both white hypertensive and black rats have increased levels of noradrenaline and 5-hydroxytryptamine in some regions of the brain (Table 2). The total tissue indole concentrations were also increased, suggesting increased levels of 5 -hydroxyindoleacetic acid, the principal metabolite of 5-hydroxytryptamine. Levels of normetanephrine, dopamine, 3-methoxytyramine, and homovanillic acid showed no differences between the groups.

The blood pressure of rats with a renal artery constriction rose steadily during the first six weeks to a stable hypertensive level. The two control groups showed no blood pressure change. No change in either Y-runway activities or nocturnal activity that could be correlated with change in blood pressure was observed.

\section{Oiscussion}

The present results confirm the earlier findings of Griew (1966) that genetic hypertensive rats show reduced spontaneous nocturnal activity. Griew also showed that hypertensive and normotensive rats had a similar response to the open field test; our results are essentially similar. The increased defecation rate may suggest an increased "emotionality" in the hypertensive rat. Conversely, the black rats had a reduced defecation rate; this is consistent with the reported quieting effect of the black gene (Keeler, 1947).

The brain noradrenaline levels of genetic hypertensive animals has been shown (Robertson \& Laverty, 1967) to be higher than in normotensive animals; in the present results, an increased level of 5-hydroxytryptamine and its metabolites has also been shown. The black rats were also found to have higher noradrenaline and 5-hydroxyindole levels than normotensive white animals.

From the present experiments, it cannot be established that the increased brain amine levels are correlated with blood pressure, since both the black rats, which are normotensive, and the hypertensive white strain have raised amine levels. The amine levels do not appear to relate to the emotionality of the animal, as expressed by its defecation rate in a strange environment, since black and hypertensive white rats have totally different defecation rates. The only possible correlation is between increased brain amines and reduced nocturnal activity in young animals.

The effects on behavior are not due to the hypertension as such, since the induction of renal hypertension is not accompanied by changes in $\mathrm{Y}$-runway behavior or nocturnal activity; brain noradrenaline levels in renal hypertensive rats are also normal (Shaepdryver, Taquini, Bernard, \& Heymans, 1963; Melville, Hodge, \& Smirk, 1966).

\section{References}

ASHCROFT, G. W., \& SHARMAN, D. F. Drug-induced changes in the concentration of 5-OR indolyl compounds in cerebrospinal fluid and caudate nucleus. Brit. J. Pharmacol, 1962, 19, 153-160.

GALLAGHER, D. J. A., \& GRIMWOOD, L. H. Simple electrical method for measuring systolic blood pressure in the intact rat. J. Physiol, 1953, 121, 163-166.

GRIEW, S. Ageing and behaviour in genetically-hypertensive rats. Proc. 7th Inth Congr. Geront., Vienna, 1966, 141-144.

JUORIO, A. V., SHARMAN, D. F., \& TRAJKOV, T. The effects of drugs on the homovanillic acid content of the corpus striatum of some rodents, Brit. J. Pharmacol, 1966, 26, 385-392.

KEELER, C. E. The association of the black (non-agouti) gene with behaviour in the Norway rat. J. Hered., 1942, 33, 371-384.

LAVERTY, R., \& TAYLOR, K. M. An improved technique for the fluorimetric estimation of catecholamines and related compounds. Proc. Univ. Otago med. Sch, 1967, 45, 8-11.

MELVILLE, A., HODGE, J. V., \& SMIRK, F. H. Tissue catecholamine levels in normotensive, genetic hypertensive and renal hypertensive rats. Proc. Univ. Otago med. Sch, 1966, 44, 17-19.

ROBERTSON, A., \& LAVERTY, R. Brain catecholamines in normotensive and hypertensive rats. Proc. Univ. Otago med. Sch, 1967, 45, 1-3.

SHAEPDRYVER, A. F. de, TAQUINI, A. C., BERNARD, P., \& HEYMANS, $C$. Tissue catecholamines in chronic renal hypertension. Arch. int. Pharmacodyn., 1963, 142, 260-266.

SMIRK, F. H., \& HALL, W. H. Inherited hypertension in rats. Nature, 1958, 182, 727-728.

STEINBERG, H., RUSHTON, R., \& TINSON, C. Modification of the effects of an amphetamine-barbiturate mixture by the past experience of rats. Nature, 1961, 192, 533-535.

\section{Note}

1. This work was financed by the Golden Kiwi Medical Research Distribution Committee. 